\title{
PARAMETRIZATIONS FOR FAMILIES OF ECM-FRIENDLY CURVES
}

\author{
ALEXANDRE GÉLIN, THORSTEN KLEINJUNG, AND ARJEN K. LENSTRA
}

\begin{abstract}
We provide a new family of elliptic curves that results in a one to two percent performance improvement of the elliptic curve integer factorization method. The speedup is confirmed by extensive tests for factors ranging from 15 to 63 bits.
\end{abstract}

\section{INTRODUCTION}

The elliptic curve method (ECM) for integer factorization was introduced by H.W. Lenstra in 1985 and published two years later in [9]. It is the asymptotically fastest method that has been published for finding relatively small factors of large composites. Although the number field sieve [8] is the most efficient general algorithm for integer factorization, there are two common use cases for ECM: it is widely used in attempts to find factors of large composites for which no information is available about the sizes of the prime factors (R. Propper found the largest ECM factor so far, a 274-bit factor of $7^{337}+1$ ) and it is used for the so-called cofactoring step of the number field sieve (where many relatively small composites have to be factored).

Given an odd composite integer $N$ to be factored, ECM performs arithmetic operations on elliptic curves considered to be defined over the finite field $\mathbf{F}_{p}$ of cardinality $p$, for an unknown prime divisor $p$ of $N$. It may find $p$ if the cardinality of at least one of these curves over $\mathbf{F}_{p}$ is smooth. For this reason, curves are used that are known to have favorable smoothness properties, such as a large torsion group over $\mathbf{Q}$ or a cardinality that is divisible by a fixed factor. Constructions of ECM-friendly curves were published by Suyama [11] (with a slight improvement by Montgomery in [10, Section 10.3.2]), Atkin-Morain [1], and generalized by Bernstein et al. in [4].

Originally formulated in [9] using Weierstrass curves, until around 2008 implementations of ECM mostly used Montgomery's approach from [10]. With the introduction of Edwards curves [6], a number of follow-up papers by Bernstein et al. [3, 5] ultimately led to " $a=-1$ twisted Edwards" curves by Hisil et al. [7] with torsion group isomorphic to $\mathbf{Z} / 2 \mathbf{Z} \times \mathbf{Z} / 4 \mathbf{Z}$ as one of the current most efficient ways to implement ECM, as shown by Bernstein et al. in [4]. For these curves, Barbulescu et al. in [2] identify three families that all have the same larger smoothness probability and an even better fourth family. In [2] a parametrization is provided for one of the three equivalent families; the others are only illustrated by - a finite set of small values found by enumeration. In particular a parametrization of the fourth and best family, which could lead to a better choice of curves for ECM, has so far not been published. By parametrization we mean that an elliptic curve along with a non-torsion point is determined as a function of some parameter: in this paper 
the parameter may be a point on some other elliptic curve or a rational number, thus giving rise to elliptic and rational parametrizations.

After a brief history in Section 2 on the use of Edwards curves in ECM, we present in Section 3 parametrizations for all families from [2] that remained to be parameterized. In Section 4 we compare our new curves to the ones used so far, observing that the curves derived from a same parametrization have similar behavior and that the fourth and best family indeed seems to lead to the best performance known for ECM. The resulting speed up is modest but worthwhile: given how much computing time is invested in ECM, the resulting practical savings could be considerable.

\section{EdWARds CURVES AND ELliptic CURVE MEthod}

Twisted Edwards curves. Let $\mathbf{K}$ be a field of characteristic different from 2 . Edwards curves are defined in [6] by equations of the form $x^{2}+y^{2}=1+d x^{2} y^{2}$, for $d \in \mathbf{K}$ with $d(d-1) \neq 0$. To enlarge the set of curves, the equation was generalized in [3] to twisted Edwards curves $a x^{2}+y^{2}=1+d x^{2} y^{2}$ for $a, d \in \mathbf{K}$ with $a d(a-d) \neq 0$. Because of the favorable properties of the arithmetic in the curve group (speed and no exception for doubling) twisted Edwards curves gained interest in applications.

Torsion group. For each curve, stage 1 of ECM attempts to compute a scalar multiple of some initial point on the curve, for a scalar equal to the product of all prime powers up to some bound and where the computation is done modulo $N$. If the order of the initial point happens to be smooth modulo at least one but not all prime factors of $N$, an inversion failure modulo $N$ reveals a factor of $N$. Because the torsion group $E_{\text {tors }}$ of the curve over $\mathbf{Q}$ injects in the curve modulo each prime that has good reduction, in ECM it helps to choose curves that have a large $E_{\text {tors }}$. The largest gain that can be obtained in this manner is modest, because according to Mazur's theorem $E_{\text {tors }}$ is isomorphic to

$$
\mathbf{Z} / n \mathbf{Z} \text { with } 1 \leq n \leq 10 \text { or } n=12 \text {, or } \mathbf{Z} / 2 \mathbf{Z} \times \mathbf{Z} / 2 n \mathbf{Z} \text { with } 1 \leq n \leq 4 .
$$

The two most profitable possibilities, $\mathbf{Z} / 12 \mathbf{Z}$ and $\mathbf{Z} / 2 \mathbf{Z} \times \mathbf{Z} / 8 \mathbf{Z}$, are characterized for families of Edwards curves in [5, Section 6]. On the other hand, the fastest scalar multiplication is obtained in [7] for $a=-1$ twisted Edward curves; as shown in [5], however, this limits the possibilities for interesting torsion groups (i.e., with cardinality greater than four) to

$$
\mathbf{Z} / 6 \mathbf{Z}, \mathbf{Z} / 8 \mathbf{Z} \text { or } \mathbf{Z} / 2 \mathbf{Z} \times \mathbf{Z} / 4 \mathbf{Z}
$$

thereby in particular excluding the two most profitable ones. For ECM the issue was settled in [4] where $a=-1$ twisted Edwards curves were compared to curves with $E_{\text {tors }}$ isomorphic to $\mathbf{Z} / 12 \mathbf{Z}$ and $\mathbf{Z} / 2 \mathbf{Z} \times \mathbf{Z} / 8 \mathbf{Z}$ : it was found that the disadvantage of the formers' smaller torsion groups is outweighed by their faster scalar multiplication.

Curves with on average higher torsion modulo $p$. Barbulescu et al. in [2] further develop $a=-1$ twisted Edwards curves with torsion group isomorphic to $\mathbf{Z} / 2 \mathbf{Z} \times \mathbf{Z} / 4 \mathbf{Z}$. In [4] these are shown to be the curves $-x^{2}+y^{2}=1-e^{4} x^{2} y^{2}$ with $e \notin\{0, \pm 1\}$. Compared to generic $e$-values and averaging over the primes $p$ of good reduction, [2] uses Galois properties to identify four families of $e$-values 
with increased average exponent of the prime 2 in the torsion group of the curve modulo $p$ : for families

$$
(i): e=g^{2} ; \quad(i i): e=\frac{g^{2}}{2}, \quad \text { and } \quad(i i i): e=\frac{2 g^{2}+2 g+1}{2 g+1}
$$

the average exponent of the prime 2 increases by $\frac{1}{6}$ from $\frac{14}{3}$ to $\frac{29}{6}$ and for family

$$
(i v): e=\frac{g-\frac{1}{g}}{2}
$$

it increases by $\frac{2}{3}$ from $\frac{14}{3}$ to $\frac{16}{3}$.

As an example, although the cardinality of the torsion group over $\mathbf{Q}$ equals eight, it is shown in [2] that for the best case (family $(i v)$ ) the cardinality of the torsion group of the curve modulo $p$ is divisible by 16 , and by 32 if $p \equiv 1 \bmod 4$ and $g(g-1)(g+1)$ is a quadratic residue modulo $p$.

Usage of these curves in ECM requires an easy way to generate them, along with an appropriate initial point on each curve. Earlier and new ways to do this are discussed in the next section.

\section{Parametrizations for CuRves With higher torsion modulo $p$}

In this section we are exclusively interested in the generation of $a=-1$ twisted Edwards curves with torsion group isomorphic to $\mathbf{Z} / 2 \mathbf{Z} \times \mathbf{Z} / 4 \mathbf{Z}$. The first known parametrization for the general case of such curves is the elliptic parametrization from [4, Theorem 3.3]; it generates a target curve (and a point of infinite order on it) as a function of a point on another elliptic curve of positive rank. Our goal is to find elliptic parametrizations for all four families $(i)-(i v)$ in (2.1) and (2.2).

For family $(i)$ this has already been done in [2]. First a new rational parametrization for the general case was derived, i.e., a function from $\mathbf{Q}$ - with finitely many exceptions - to $a=-1$ twisted Edwards curves with torsion group isomorphic to $\mathbf{Z} / 2 \mathbf{Z} \times \mathbf{Z} / 4 \mathbf{Z}$, along with a point of infinite order on each curve. It was then checked if a special choice of the rational parameters leads to curves with $e$-values as in (2.1) or (2.2). That turned out to work for family $(i)$, but due to limitations imposed by the rational parametrization it failed for families (ii)-(iv).

In this section we review the approach from [2], and we present the results of a search that we conducted for new rational parametrizations for the general case. The newly found rational parametrizations turn out to suffice for our purpose: following the approach from [2] it is shown that special choices of the rational parameters lead to elliptic parametrizations for all four families $(i)-(i v)$.

General conditions. From the equation $-x^{2}+y^{2}=1-e^{4} x^{2} y^{2}$ with $e \notin\{0, \pm 1\}$ for the general case, it follows that $e$ and $-e$ lead to the same curve. Considering the variable change $(x, y) \longmapsto\left(x e^{2}, \frac{1}{y}\right)$, the curves for $e$ and $\frac{1}{e}$ are birationally equivalent. Thus, only one element of $\left\{ \pm e, \pm \frac{1}{e}\right\}$ is of interest and we fix $e>1$.

Furthermore, as we require points of infinite order, the curves must have positive rank and easily identifiable torsion points in order to be able to avoid them. The latter can be done, because

- $(0,1)$ is the neutral element,

- $(0,-1)$ and $\left(\infty, \pm \frac{1}{e^{2}}\right)$ are the three 2-torsion points, and

- $\left( \pm \frac{1}{e}, \pm \frac{1}{e}\right)$ are the four 4 -torsion points. 
A point $(x, y)$ on the curve is therefore a non-torsion point if and only if $x \notin\{0, \infty\}$ and $x e \neq \pm 1$.

Earlier parametrizations. As mentioned above, the first parametrization for the general case, from [4, Theorem 3.3], works by selecting a particular elliptic curve over $\mathbf{Q}$ of positive rank and by showing how a rational point on it provides an $a=-1$ twisted Edwards curve with torsion group isomorphic to $\mathbf{Z} / 2 \mathbf{Z} \times \mathbf{Z} / 4 \mathbf{Z}$, along with a non-torsion point. The first rational parametrization generalizes this approach. It is [2, Theorem 3.7]:

Theorem 3.1. For nonzero $t \in \mathbf{Q} \backslash\left\{ \pm 1, \pm 3^{ \pm 1}\right\}$ let

$e_{1}=\frac{3\left(t^{2}-1\right)}{8 t}, x_{1}=\frac{1}{4 e_{1}^{3}+3 e_{1}}=\frac{128 t^{3}}{27 t^{6}+63 t^{4}-63 t^{2}-27}$ and $y_{1}=\frac{9 t^{4}-2 t^{2}+9}{9 t^{4}-9}$.

Then $\left(x_{1}, y_{1}\right)$ is a non-torsion point on the $a=-1$ twisted Edwards curve $-x^{2}+y^{2}=$ $1-e_{1}^{4} x^{2} y^{2}$ with torsion group isomorphic to $\mathbf{Z} / 2 \mathbf{Z} \times \mathbf{Z} / 4 \mathbf{Z}$.

Proof. The excluded $t$-values imply that $e \notin\{0, \pm 1\}$ and that $x_{1}$ and $y_{1}$ are welldefined. The point $\left(x_{1}, y_{1}\right)$ can be seen to be on the curve and it is a non-torsion point because $x_{1} \notin\{0, \infty\}$ and $x_{1} e_{1} \neq \pm 1$.

Compared to the parametrization from [4, Theorem 3.3], the above rational parametrization reduces the computation required for curve generation, and the simple formula for $e$ facilitates the search for $t$-values that lead to an $e$-value that satisfies (2.1) or (2.2). It is easy to check if a rational $e$-value belongs to family $(i)$ or $(i i)$. Families $(i i i)$ and $(i v)$ look more cumbersome, but can easily be dealt with using the following alternative characterizations.

- Because $e=\frac{2 g^{2}+2 g+1}{2 g+1}$ if and only if $2 g^{2}+2(1-e) g+(1-e)=0$ and because a rational $g$ satisfying the latter polynomial exists if and only if the discriminant $4\left(e^{2}-1\right)$ is a rational square, it follows that

$$
e=\frac{2 g^{2}+2 g+1}{2 g+1} \Longleftrightarrow e^{2}-1 \text { is a square. }
$$

- Arguing identically (which results in discriminant $4\left(e^{2}+1\right)$ ) it follows that

$$
e=\frac{g-\frac{1}{g}}{2} \Longleftrightarrow e^{2}+1 \text { is a square. }
$$

Corollary 3.2. (This is [2, Corollary 3.8].) Consider the elliptic curve $y^{2}=$ $x^{3}-36 x$ of rank one, with the point $(-3,9)$ generating a non-torsion subgroup. For any point $(x, y)$ on this curve and

$$
t=\frac{x+6}{x-6}
$$

the $a=-1$ twisted Edwards curve with torsion group isomorphic to $\mathbf{Z} / 2 \mathbf{Z} \times \mathbf{Z} / 4 \mathbf{Z}$ defined as in Theorem 3.1 belongs to family $(i)$ and has positive rank over $\mathbf{Q}$.

Proof. This follows from

$$
e_{1}=\frac{3\left(t^{2}-1\right)}{8 t}=\frac{9 x}{x^{2}-36}=\left(\frac{3 x}{y}\right)^{2}
$$

and the fact that $\left(x_{1}, y_{1}\right)$ is a non-torsion point. 
Remark 3.3. Corollary 3.2 provides an elliptic parametrization for family (i). Attempting this approach for the other three families gives rise to curves of rank zero, and thus not the infinite families desired.

New rational parametrizations. To find new infinite families of curves, we rewrite the general case equation $-x^{2}+y^{2}=1-e^{4} x^{2} y^{2}$ as

$$
y^{2}=\frac{1+x^{2}}{1+e^{4} x^{2}} \text {. }
$$

The resulting condition that the right hand side is a square is equivalent to requiring that $\left(1+x^{2}\right)\left(1+e^{4} x^{2}\right)=e^{4} x^{4}+\left(1+e^{4}\right) x^{2}+1$ is a square. With $x=\frac{u}{v}$ we get the equivalent condition that for the chosen values of $e, u$, and $v$ the polynomial $e^{4} u^{4}+\left(1+e^{4}\right) u^{2} v^{2}+v^{4}$ must evaluate to a square.

Choosing $u$ and $v$ as polynomials in $e$, we are interested in the square-free part of the resulting polynomial in $e$ and in $e$-values for which the square-free part evaluates to a rational square. A square-free part of degree higher than four corresponds to a hyperelliptic curve that is known to have a finite number of rational points; because we are interested in infinite families of curves, this case is of no interest to us.

The following search was conducted (using PARI/GP [12] for the polynomial factorizations):

- $x=u^{ \pm 1}$ for all integer polynomials $u$ of degree at most two and coefficients absolutely bounded by 100, and of degree at most four and coefficients absolutely bounded by ten;

- $x=\frac{u}{v}$ for all integer polynomials $u, v$ of degree at most three and coefficients absolutely bounded by ten.

The only square-free part of degree two thus found leads to the rational parametrization from Theorem 3.1. All other square-free parts have degree equal to four, and thus each corresponds to an elliptic curve. Each one that has positive rank then leads to an elliptic parametrization (i.e., curves as functions of points on some other elliptic curve), but as we set out to find rational parametrizations (i.e., curves as functions of rational numbers) this is not what we are interested in.

To address this we "manually" searched through the polynomials attempting to find commonalities among the relevant square-free parts, which ultimately resulted in parameterized infinite families of curves. Given such an infinite curve parametrization (parameterized by $k$ in the example below), it suffices to parameterize a finite number of points per curve. Our approach essentially consists in identifying these families from the derived small values and check if they lead to good subfamilies.

Example 3.4. To illustrate this strategy, substituting $\frac{e+k}{k e-1}$ for $x$ in Equation (3.1), it is found that $\frac{k^{2}+1}{e^{4}+2 k e^{3}+\left(k^{2}-1\right) e^{2}-2 k e+1}$ must be a square. With the fixed point $e=\frac{3}{4 k}$ the denominator becomes $\left(\frac{4 k^{2}+9}{16 k^{2}}\right)^{2}$ so that for each $k$ for which $k^{2}+1$ is a square, we have a point $(x, y)$ satisfying the curve equation $-x^{2}+y^{2}=1-\left(\frac{3}{4 k}\right)^{4} x^{2} y^{2}$ for the general case. This results in infinitely many such curves.

Using several families of degree four polynomials (and, for some, different points on the same curve), this resulted in the six additional new rational parametrizations in Theorem (3.5), the proof of which is identical to the proof of Theorem 3.1. For completeness the earlier result from Theorem 3.1 is included. 
Theorem 3.5. For $1 \leq j \leq 7$ and for each nonzero $t \in \mathbf{Q} \backslash S_{j}$ the point $\left(x_{j}, y_{j}\right)$ is a non-torsion point on the $a=-1$ twisted Edwards curve $-x^{2}+y^{2}=1-e_{j}^{4} x^{2} y^{2}$ with torsion group isomorphic to $\mathbf{Z} / 2 \mathbf{Z} \times \mathbf{Z} / 4 \mathbf{Z}$ :

\begin{tabular}{ccccc}
$j$ & $S_{j}$ & $e_{j}$ & $x_{j}$ & $y_{j}$ \\
\hline 1 & $\left\{ \pm 1, \pm 3^{ \pm 1}\right\}$ & $\frac{3\left(t^{2}-1\right)}{8 t}$ & $\frac{128 t^{3}}{27 t^{6}+63 t^{4}-63 t^{2}-27}$ & $\frac{9 t^{4}-2 t^{2}+9}{9 t^{4}-9}$ \\
2 & $\{-2,-1, \pm 4\}$ & $\frac{t^{2}+2 t+4}{2 t+2}$ & $\frac{2 t^{3}+2 t^{2}-8 t-8}{t^{4}+6 t^{3}+12 t^{2}+16 t}$ & $\frac{2 t^{5}+14 t^{4}+40 t^{3}+44 t^{2}+32 t+16}{t^{6}+4 t^{5}+10 t^{4}+20 t^{3}+40 t^{2}+64 t+64}$ \\
3 & $\{ \pm 2\}$ & $\frac{t^{2}+4}{3 t}$ & $\frac{12 t^{2}-24}{t^{4}-4 t^{2}-32}$ & $\frac{3 t^{6}-12 t^{4}+120 t^{2}}{5 t^{6}+12 t^{4}+128}$ \\
4 & $\{-2,-1, \pm 4\}$ & $\frac{t^{2}+4 t}{t^{2}-4}$ & $\frac{2 t^{3}+2 t^{2}-8 t-8}{t^{4}+6 t^{3}+12 t^{2}+16 t}$ & $\frac{t^{6}+6 t^{5}+10 t^{4}-16 t^{3}-48 t^{2}-32 t-32}{t^{6}+6 t^{5}+10 t^{4}+16 t^{3}+48 t^{2}+64 t}$ \\
5 & $\{ \pm 4, \pm 8\}$ & $\frac{4 t^{4}-1024}{t^{5}+512 t}$ & $\frac{96 t^{6}+49152 t^{2}}{t^{8}-1280 t^{4}+262144}$ & $\frac{t^{12}+3840 t^{8}+1966080 t^{4}+134217728}{t^{12}-768 t^{8}+786432 t^{4}-167772160}$ \\
6 & $\{ \pm 1, \pm 2, \pm 4\}$ & $\frac{t^{3}+8 t}{4 t^{2}+8}$ & $\frac{12 t^{2}+24}{t^{4}+4 t^{2}-32}$ & $\frac{4 t^{6}+24 t^{4}+192 t^{2}+320}{5 t^{6}+48 t^{4}+96 t^{2}+256}$ \\
7 & $\{ \pm 2\}$ & $\frac{t^{3}-8 t}{4 t^{2}-8}$ & $\frac{12 t^{2}-24}{t^{4}-4 t^{2}-32}$ & $\frac{4 t^{6}-24 t^{4}+192 t^{2}-320}{5 t^{6}-48 t^{4}+96 t^{2}-256}$
\end{tabular}

Imposing the aforementioned conditions to obtain all four families $(i)-(i v)$ (as mentioned above, it suffices to test if $\left\{e, 2 e, e^{2} \pm 1\right\}$ contains a square) leads to the corollary below. For completeness it includes the result of Corollary 3.2.

Corollary 3.6. For $1 \leq j \leq 4$ let $\left(e_{j}, x_{j}, y_{j}\right)$ be functions of $t$ as in Theorem 3.1. For each case below the elliptic curve $E$ has rank one, torsion group consisting of the set $T$ adjoined with the neutral element, and non-torsion point $Q$, and for each point $(x, y)$ on $E$ the pair $\left(x_{j}, y_{j}\right)$ is a non-torsion point on the $a=-1$ twisted Edwards curve $-x^{2}+y^{2}=1-e_{j}^{4} x^{2} y^{2}$ with torsion group isomorphic to $\mathbf{Z} / 2 \mathbf{Z} \times \mathbf{Z} / 4 \mathbf{Z}$ of positive rank and of the family listed. The last two columns list the $e_{j}$-value of the first curve constructed along with the non-torsion point.

\begin{tabular}{|c|c|c|c|c|c|c|c|}
\hline family & $j$ & $E$ & $T$ & $Q$ & $t$ & & $\begin{array}{l}\text { e generated by } Q: \\
\text { non-torsion point }\end{array}$ \\
\hline (i) & 1 & $y^{2}=x^{3}-36 x$ & $\{(0,0),( \pm 6,0)\}$ & $(-3,9)$ & $\frac{x+6}{x-6}$ & $\frac{16}{9}$ & $\left(\frac{12}{91}, \frac{27}{29}\right)$ \\
\hline (ii) & 2 & $y^{2}=x^{3}+3 x$ & $\{(0,0)\}$ & $(1,2)$ & $x-1$ & $\frac{49}{8}$ & $\left(\frac{440}{1911}, \frac{15688}{132937}\right)$ \\
\hline$(i i)$ & 3 & $y^{2}=x^{3}+9 x$ & $\{(0,0)\}$ & $(4,10)$ & $\frac{2 x}{3}$ & $\frac{25}{18}$ & $\left(\frac{48}{575}, \frac{15579}{15725}\right)$ \\
\hline (iii) & 3 & $y^{2}=x^{3}-x^{2}-64 x+64$ & $\{(1,0),( \pm 8,0)\}$ & $(-6,14)$ & $\frac{8 x-8}{y}$ & $\frac{5}{3}$ & $\left(\frac{21}{20}, \frac{87}{185}\right)$ \\
\hline$(i i i)$ & 4 & $y^{2}=x^{3}-12 x$ & $\{(0,0)\}$ & $(-2,4)$ & $\frac{x-2}{2}$ & $\frac{65}{56}$ & $\left(\frac{252}{3965}, \frac{444976}{445705}\right)$ \\
\hline$(i v)$ & 4 & $y^{2}=x^{3}-x^{2}-9 x+9$ & $\{(1,0),( \pm 3,0)\}$ & $(5,8)$ & $\frac{4 x+4}{y-4}$ & $\frac{15}{8}$ & $\left(\frac{28}{195}, \frac{3152}{3495}\right)$ \\
\hline
\end{tabular}

Proof. With the proof of Corollary 3.2 and the characterizations given before it, the verifications in the table below suffice.

\begin{tabular}{lll} 
family & $j$ & \\
\cline { 1 - 2 }$($ ii $)$ & $2:$ & $e_{2}=\frac{t^{2}+2 t+4}{2 t+2}=\frac{x^{2}+3}{2 x}=\frac{1}{2}\left(\frac{y}{x}\right)^{2}$ \\
$($ ii $)$ & $3:$ & $e_{3}=\frac{t^{2}+4}{3 t}=\frac{2 x^{2}+18}{9 x}=\frac{1}{2}\left(\frac{2 y}{3 x}\right)^{2}$ \\
$($ iii $)$ & $3:$ & $e_{3}^{2}-1=\frac{x^{4}-4 x^{3}+132 x^{2}-256 x+4096}{36 y^{2}}=\left(\frac{x^{2}-2 x+64}{6 y}\right)^{2}$ \\
$($ iii $)$ & $4:$ & $e_{4}^{2}-1=\frac{16 x^{3}-192 x}{x^{4}-8 x^{3}-8 x^{2}+96 x+144}=\left(\frac{4 y^{2}}{x^{2}-4 x-12}\right)^{2}$ \\
$($ iv $)$ & $4:$ & $e_{4}^{2}+1=\left(\frac{x^{4}+4 x^{3}+14 x^{2}-108 x+153}{x^{4}-4 x^{3}-18 x^{2}-16 x y+12 x+48 y+9}\right)^{2}$
\end{tabular}


Remark 3.7. Two of the curves in the $e_{j}$-column of the table in Corollary 3.6 already appeared in [4, Table 3.1], namely those in the first and fourth row (though for the latter [4] used the different non-torsion point $\left.\left(\frac{27}{11}, \frac{5}{13}\right)\right)$.

The search that led to Corollary 3.6 did not identify any elliptic curve of positive rank for $j>4$, and no other elliptic curve of positive rank for $j \leq 4$ either.

\section{EFFECTIVEnESS OF THE NEW CURVES}

In this section we consider the effectiveness of our new curves when used in ECM and compare them to the curves proposed for ECM in [4]. To facilitate the comparison we conduct the same tests as in [4] and borrow some of their notation.

Earlier work. In [4] the effectiveness of Edwards curves for ECM was investigated. For each of the five torsion groups (isomorphic to $\mathbf{Z} / 6 \mathbf{Z}, \mathbf{Z} / 8 \mathbf{Z}, \mathbf{Z} / 12 \mathbf{Z}, \mathbf{Z} / 2 \mathbf{Z} \times$ $\mathbf{Z} / 4 \mathbf{Z}$, and $\mathbf{Z} / 2 \mathbf{Z} \times \mathbf{Z} / 8 \mathbf{Z}$ ) a set of a thousand Edwards curves was generated as described in [4], with $a=-1$ when possible (i.e., for the three smallest torsion groups). For each of the 5000 resulting curves the EECM software from [5] was applied to all $b$-bit primes for $15 \leq b \leq 26$ (with ECM bounds depending on the targeted $b$-bit prime as in [5]). For each curve and each $b$ the number of $b$-bit primes found (the "yield") was tallied, with the resulting counts extensively detailed in five very informative tables. Here a prime $p$ is said to be "found" by a curve if the cardinality of the curve over $\mathbf{F}_{p}$ is smooth with respect to the ECM bounds used.

On average the curves with torsion group isomorphic to $\mathbf{Z} / 6 \mathbf{Z}$ performed best, because of the relatively large number of primes found and because $a=-1$ allows fast scalar multiplication. Among these, a particularly good curve is $-x^{2}+y^{2}=$ $1-\frac{13312}{18225} x^{2} y^{2}$, identified by the non-torsion point $\left(\frac{825}{2752}, \frac{1521}{1504}\right)$ in the \#1-column (for $b=21$ and $b \geq 24$ ) and \#2-column (for $b=19,22$ ) of [4, Table 5.1]. We re-derived its tallies for the $\mathcal{C}_{6}$-column of Table 1 (six figures of which thus already appeared in [4, Table 5.1]). Another interesting result was that among the curves with torsion group isomorphic to $\mathbf{Z} / 2 \mathbf{Z} \times \mathbf{Z} / 4 \mathbf{Z}$ four curves appeared to stand out. Indeed, these four curves happened to belong to the families with favorable Galois properties that were identified in [2]: two of family $(i)$ and one each of families (iii) and $(i v)$ and in $\left[4\right.$, Table 3.1] identified by the non-torsion points $\left(\frac{12}{91}, \frac{27}{29}\right),\left(\frac{3}{14}, \frac{1}{17}\right)$, $\left(\frac{27}{11}, \frac{5}{13}\right)$, and $\left(\frac{12}{343}, \frac{1404}{1421}\right)$, respectively. The latter one "easily outperforms" the other 999 curves for $b \geq 19$, the reason of which is not identified in [2] and which we now know to be due to the fact that it is of family $(i v)^{1}$ : in [4, Table 3.1] it is "best" for $b=17$ and $b \geq 19$ and "second best" for $b=16,18$. This curve has equation $-x^{2}+y^{2}=1-\left(\frac{77}{36}\right)^{4} x^{2} y^{2}$ and does not appear in our parametrization of curves of family $(i v)$. Its re-derived tallies are listed in the $\mathcal{C}_{2 \times 4}$-column of Table 1 (all figures of which except for $b=15$ thus already appeared in the \#1 or \#2-column of $[4$, Table 3.1]).

Comparison to earlier work. For one hundred curves of family (iv) as parameterized in the last row of the table in Corollary 3.6 we ran the same tests as in [4] with the same EECM software and parameters. The curves we used are denoted by $\mathcal{C}_{[m]}$ for $1 \leq m \leq 100$, where $\mathcal{C}_{[m]}$ is constructed from the point $(x, y)=m Q$ with $Q=(5,8)$ on the curve $y^{2}=x^{3}-x^{2}-9 x+9$. The \#1 and \#100-column in Table 1 list the largest and smallest, respectively, number of primes found per

\footnotetext{
${ }^{1}$ This was recognized by Peter L. Montgomery.
} 
curve, with the average-column averaging the counts over all one hundred curves. Results of the tests for families $(i)-($ iii) are less interesting and not reported.

Per $b$-value the ratio of the best yield over $\mathcal{C}_{[1]}, \mathcal{C}_{[2]}, \ldots, \mathcal{C}_{[100]}$ and the yield of the single best performing earlier curve $\mathcal{C}_{6}$ is given in the $\# 1 / \mathcal{C}_{6}$-column of Table 1 , but no single new curve has been identified that actually realizes the small gain suggested (but see Table 2 below). Indeed, the outcome of the same performance comparison between the average of the $\mathcal{C}_{[m]^{-}}$-curves and $\mathcal{C}_{6}$ is more variable and with 65 of the one hundred $\mathcal{C}_{[m]}$-curves having a higher average yield than $\mathcal{C}_{6}$, the performance is close. The curve with the best average ratio (of 1.0064) compared to $\mathcal{C}_{6}$ is $\mathcal{C}_{[93]}$, and curves $\mathcal{C}_{\text {[22] }}$ and $\mathcal{C}_{\text {[86] }}$ are the only two curves that have higher yield than $\mathcal{C}_{6}$ (and thus than $\mathcal{C}_{2 \times 4}$ ) for all but three $b$-values; there are ten $\mathcal{C}_{[m]}$ curves for which the yield is lower than for $\mathcal{C}_{6}$ for four $b$-values. As can be seen in Table 1 , for $b=23$ all $\mathcal{C}_{[m]}$-curves considered have higher yield than $\mathcal{C}_{6}$. Unlike [4], we do not specify which of $\mathcal{C}_{[1]}, \mathcal{C}_{[2]}, \ldots, \mathcal{C}_{[100]}$ has the best yield because with no curve appearing more than twice among the "top three" this information is useless. This is illustrated, in the ${ }^{\# 1} / \# 100^{-c o l u m n}$, by the ratio of the yields of the best and worst performing $\mathcal{C}_{[m]}$ per $b$-value: with small ratios all $\mathcal{C}_{[m]}$-curves tested can be seen to behave similarly. The figures in the ratio-columns of the tables in [4] are much larger - with one thousand curves per table they not only cast their net much wider, but they also allow a greater variation (of Galois properties) of curves per batch.

TABLE 1. Yields for $\mathcal{C}_{6}$ and the family $(i v)$ curves $\mathcal{C}_{2 \times 4}, \mathcal{C}_{[1]}, \mathcal{C}_{[2]}, \ldots, \mathcal{C}_{[100]}$.

\begin{tabular}{|c|c|c|c|c|c|c|c|}
\hline$b$ & $\mathcal{C}_{6}$ & $\mathcal{C}_{2 \times 4}$ & $\begin{array}{c}\# 1 \\
\text { (among }\end{array}$ & $\begin{array}{c}\text { average } \\
\mathcal{C}_{[1]}, \mathcal{C}_{[2]}, \ldots\end{array}$ & $\begin{array}{r}\# 100 \\
\left., \mathcal{C}_{[100]}\right) \\
\end{array}$ & $\# 1 / \mathcal{C}_{6}$ & $\# 1 / \# 100$ \\
\hline 15 & 1127 & 1049 & 1202 & 1155.36 & 1103 & 1.0665 & 1.0897 \\
\hline 16 & 1693 & 1564 & 1806 & 1737.32 & 1664 & 1.0667 & 1.0853 \\
\hline 17 & 3299 & 2985 & 3324 & 3197.86 & 3077 & 1.0075 & 1.0802 \\
\hline 18 & 6150 & 5529 & 6168 & 6020.01 & 5921 & 1.0029 & 1.0417 \\
\hline 19 & 10802 & 10200 & 10881 & 10723.75 & 10500 & 1.0073 & 1.0362 \\
\hline 20 & 16148 & 15486 & 16396 & 16197.71 & 15955 & 1.0153 & 1.0276 \\
\hline 21 & 24160 & 22681 & 24312 & 24003.34 & 23655 & 1.0062 & 1.0277 \\
\hline 22 & 48378 & 46150 & 48894 & 48515.60 & 48114 & 1.0106 & 1.0162 \\
\hline 23 & 83339 & 82743 & 85525 & 84839.98 & 84254 & 1.0262 & 1.0150 \\
\hline 24 & 193069 & 187596 & 193558 & 192825.73 & 191961 & 1.0025 & 1.0083 \\
\hline 25 & 318865 & 311864 & 320498 & 319154.79 & 317304 & 1.0051 & 1.0100 \\
\hline 26 & 493470 & 480006 & 495082 & 493556.42 & 492364 & 1.0032 & 1.0055 \\
\hline
\end{tabular}

All our parameterized curves have higher yields than the curve $\mathcal{C}_{2 \times 4}$, even though they are all family (iv) curves. This is due to our choice of the non-torsion point $\left(x_{j}, y_{j}\right)$ which implies that the group of the curve modulo about half of the primes contains a point $P$ such that $\left(x_{j}, y_{j}\right)=2 P$, thereby for those primes effectively adding a doubling in the scalar multiplication in ECM. The effect diminishes with larger $b$-values (see also the last column of Table 2). 
Additional tests. On the EECM website ${ }^{2}$ the yields of ECM using a fixed Edwards curve are given when applied to batches of $2^{20} b$-bit primes, for $b$ up to 63 . We used the same EECM software and parameters (but our own sets of $2^{20} b$-bit primes) to conduct the same experiment for the following four Edwards curves:

- the curve $\mathcal{C}_{[1]}:-x^{2}+y^{2}=1-\left(\frac{15}{8}\right)^{4} x^{2} y^{2}$, the first curve of our parametrization of family $(\mathrm{iv})$ curves;

- the above curve $\mathcal{C}_{6}$, i.e., the best performing curve from [4];

- the curve $\mathcal{C}_{12}: x^{2}+y^{2}=1-\frac{24167}{25} x^{2} y^{2}$ from the EECM website, with torsion group of order twelve and a non-torsion point $\left(\frac{5}{23}, \frac{-1}{7}\right)$ with very small coordinates;

- the above curve $\mathcal{C}_{2 \times 4}$ from [4] belonging to family (iv), but not appearing in our parametrization of family (iv) curves.

As can be seen in Table 2 the yields are very close. The average yield-ratios of curve $\mathcal{C}_{[1]}$ compared to the three other curves (in the last row of Table 2) suggest that the new curve $\mathcal{C}_{[1]}$ overall performs best. Note too that the three other curves were chosen as the best among large batches of previously known curves, whereas $\mathcal{C}_{[1]}$ is just the first one of our newly parameterized family $(i v)$ curves. Based on the final column of Table 1, we expect that all these new curves behave similarly.

The non-monotone yield decrease, consistent among the four curves tested, can be attributed to the choice of parameters in the EECM software.

\section{Conclusion}

In [2] favorable properties of the Galois group structures of $a=-1$ twisted Edwards curves with torsion group isomorphic to $\mathbf{Z} / 2 \mathbf{Z} \times \mathbf{Z} / 4 \mathbf{Z}$ were identified. This gave rise to four families of curves that looked promising for application in ECM. A new rational parametrization of $a=-1$ twisted Edwards curves with torsion group isomorphic to $\mathbf{Z} / 2 \mathbf{Z} \times \mathbf{Z} / 4 \mathbf{Z}$ then led to an elliptic parametrization for the curves in one family, but not the most promising one.

In this paper we extended the constructions from [2] by developing six further rational parametrizations, and use three of them to formulate five new elliptic parametrizations that enable fast generation of curves for all families of curves from [2]. We conducted the same tests as described in [4] for the family of curves that are, based on their Galois properties, most promising for ECM. With respect to the criteria from [4] usage of this family of curves leads to slightly better performance of ECM than reported before, with no significant fluctuations across curves from this same family. The newly parameterized curves may prove to be most useful for ECM-based cofactoring in the number field sieve. We do not claim that our results are complete: other parametrizations than the ones presented in this paper may exist.

All potential new savings identified in this paper rely on the properties of the curves used, not on the way arithmetic on the curve is performed. Indeed, we rely on the same improvement from [7] that is exploited in [4] and use the same software that was used in [4].

\footnotetext{
$2_{\text {http: //eecm.cr.yp.to/performance.html }}$
} 
TABLE 2. Yield comparisons for $\mathcal{C}_{[1]}$ versus $\mathcal{C}_{6}, \mathcal{C}_{12}$ and $\mathcal{C}_{2 \times 4}$.

\begin{tabular}{|c|c|c|c|c|c|c|c|}
\hline$b$ & $\mathcal{C}_{[1]}$ & $\mathcal{C}_{6}$ & $\mathcal{C}_{[1]} / \mathcal{C}_{6}$ & $\mathcal{C}_{12}$ & $\mathcal{C}_{[1]} / \mathcal{C}_{12}$ & $\mathcal{C}_{2 \times 4}$ & $\mathcal{C}_{[1]} / \mathcal{C}_{2 \times 4}$ \\
\hline 27 & 263563 & 260933 & 1.0100 & 257558 & 1.0233 & 259377 & 1.0161 \\
\hline 28 & 212253 & 209813 & 1.0116 & 206965 & 1.0255 & 208819 & 1.0164 \\
\hline 29 & 179190 & 176620 & 1.0145 & 174302 & 1.0280 & 176699 & 1.0140 \\
\hline 30 & 141182 & 139827 & 1.0096 & 137953 & 1.0234 & 138984 & 1.0158 \\
\hline 31 & 197013 & 195906 & 1.0056 & 193954 & 1.0157 & 195365 & 1.0084 \\
\hline 32 & 161111 & 159685 & 1.0089 & 158323 & 1.0176 & 159552 & 1.0097 \\
\hline 33 & 129949 & 128477 & 1.0114 & 127719 & 1.0174 & 128326 & 1.0126 \\
\hline 34 & 131982 & 131397 & 1.0044 & 130116 & 1.0143 & 130511 & 1.0112 \\
\hline 35 & 131837 & 130694 & 1.0087 & 129699 & 1.0164 & 131161 & 1.0051 \\
\hline 36 & 114826 & 113772 & 1.0092 & 112689 & 1.0189 & 113775 & 1.0092 \\
\hline 37 & 103744 & 102681 & 1.0103 & 102515 & 1.0119 & 103173 & 1.0055 \\
\hline 38 & 85135 & 83839 & 1.0154 & 83778 & 1.0161 & 84120 & 1.0120 \\
\hline 39 & 73526 & 73069 & 1.0062 & 72897 & 1.0086 & 73376 & 1.0020 \\
\hline 40 & 59619 & 59265 & 1.0059 & 58970 & 1.0110 & 59955 & 0.9943 \\
\hline 41 & 83967 & 83694 & 1.0032 & 83295 & 1.0080 & 83323 & 1.0077 \\
\hline 42 & 73730 & 73739 & 0.9998 & 73653 & 1.0010 & 73249 & 1.0065 \\
\hline 43 & 60978 & 60573 & 1.0066 & 60611 & 1.0060 & 60695 & 1.0046 \\
\hline 44 & 50238 & 49714 & 1.0105 & 49509 & 1.0147 & 50077 & 1.0032 \\
\hline 45 & 44354 & 43706 & 1.0148 & 43825 & 1.0120 & 44259 & 1.0021 \\
\hline 46 & 40133 & 39754 & 1.0095 & 39385 & 1.0189 & 39873 & 1.0065 \\
\hline 47 & 46291 & 46167 & 1.0026 & 45951 & 1.0073 & 46240 & 1.0011 \\
\hline 48 & 40969 & 40569 & 1.0098 & 40683 & 1.0070 & 40703 & 1.0065 \\
\hline 49 & 37956 & 37428 & 1.0141 & 37099 & 1.0231 & 37555 & 1.0106 \\
\hline 50 & 35096 & 34963 & 1.0038 & 35421 & 0.9908 & 35202 & 0.9969 \\
\hline 51 & 29503 & 29105 & 1.0136 & 29023 & 1.0165 & 29209 & 1.0100 \\
\hline 52 & 29780 & 29191 & 1.0201 & 29342 & 1.0149 & 29666 & 1.0038 \\
\hline 53 & 27430 & 27153 & 1.0102 & 27069 & 1.0133 & 27594 & 0.9940 \\
\hline 54 & 23996 & 23667 & 1.0139 & 23896 & 1.0041 & 23649 & 1.0146 \\
\hline 55 & 25316 & 25064 & 1.0100 & 24883 & 1.0174 & 25023 & 1.0117 \\
\hline 56 & 22471 & 22255 & 1.0097 & 21828 & 1.0294 & 22411 & 1.0026 \\
\hline 57 & 20449 & 20169 & 1.0138 & 20253 & 1.0096 & 20276 & 1.0085 \\
\hline 58 & 20826 & 20313 & 1.0252 & 20303 & 1.0257 & 20578 & 1.0120 \\
\hline 59 & 18527 & 18231 & 1.0162 & 18029 & 1.0276 & 18340 & 1.0101 \\
\hline 60 & 16287 & 16016 & 1.0169 & 16021 & 1.0166 & 16306 & 0.9988 \\
\hline 61 & 13638 & 13482 & 1.0115 & 13488 & 1.0111 & 13462 & 1.0130 \\
\hline 62 & 18056 & 18083 & 0.9985 & 18071 & 0.9991 & 18351 & 0.9839 \\
\hline 63 & 15657 & 15651 & 1.0003 & 15613 & 1.0028 & 15747 & 0.9942 \\
\hline & rerages & $\mathcal{C}_{[1]} / \mathcal{C}_{6}$ & 1.0099 & $\mathcal{C}_{[1]} / \mathcal{C}_{12}$ & 1.0142 & $\mathcal{C}_{[1]} / \mathcal{C}_{2 \times}$ & : 1.0063 \\
\hline
\end{tabular}

\section{ACKNowledgements}

This work has been supported in part by the European Union's H2020 Programme under grant agreement number ERC-669891. 


\section{REFERENCES}

1. Arthur O. L. Atkin and François Morain, Finding suitable curves for the elliptic curve method of factorization, Mathematics of Computation 60 (1993), 399-405.

2. Razvan Barbulescu, Joppe W. Bos, Cyril Bouvier, Thorsten Kleinjung, and Peter L. Montgomery, Finding ECM-friendly curves through a study of Galois properties, Proceedings of the Tenth Algorithmic Number Theory Symposium, 2012, pp. 63-86.

3. Daniel J. Bernstein, Peter Birkner, Marc Joye, Tanja Lange, and Christiane Peters, Twisted Edwards curves, Progress in Cryptology - AFRICACRYPT 2008, Proceedings, 2008, pp. 389405.

4. Daniel J. Bernstein, Peter Birkner, and Tanja Lange, Starfish on strike, Progress in Cryptology - LATINCRYPT 2010, Proceedings, 2010, pp. 61-80.

5. Daniel J. Bernstein, Peter Birkner, Tanja Lange, and Christiane Peters, ECM using Edwards curves, Mathematics of Computation 82 (2013), no. 282, 1139-1179.

6. Harold M. Edwards, A normal form for elliptic curves, Bulletin of the American Mathematical Society 44 (2007), 393-422.

7. Huseyin Hisil, Kenneth Koon-Ho Wong, Gary Carter, and Ed Dawson, Twisted Edwards curves revisited, Advances in Cryptology - ASIACRYPT 2008, Proceedings, 2008, pp. 326343.

8. Arjen K. Lenstra and Hendrik W. Lenstra Jr., The development of the number field sieve, Lecture Notes in Mathematics, vol. 1554, Springer-Verlag, 1993.

9. Hendrik W. Lenstra Jr., Factoring integers with elliptic curves, Annals of Mathematics 126 (1987), 649-673.

10. Peter L. Montgomery, Speeding the Pollard and elliptic curve methods of factorization, Mathematics of Computation 48 (1987), 243-264.

11. Hiromi Suyama, Informal preliminary report, (cited in [10]), 1985.

12. The PARI Group, Bordeaux, PARI/GP version 2.7.0, 2014, available from http://pari.math.u-bordeaux.fr/.

Sorbonne Universités, UPMC PAris 6, UMR 7606, LIP6, 75005, PARis, France

E-mail address: alexandre.gelin@lip6.fr

École Polytechnique Fédérale de Lausanne, EPFL IC LACAL, Switzerland

École Polytechnique Fédérale de Lausanne, EPFL iC LACAL, Switzerland 